\title{
Improving vascular maturation using noncoding RNAs increases antitumor effect of chemotherapy
}

\author{
Lingegowda S. Mangala, ${ }^{1,2}$ Hongyu Wang, ${ }^{3,4}$ Dahai Jiang, ${ }^{1,2}$ Sherry Y. Wu, ${ }^{1}$ Anoma Somasunderam, ${ }^{5}$ \\ David E. Volk, ${ }^{3,4}$ Ganesh L. R. Lokesh, ${ }^{3}$ Xin Li, ${ }^{3}$ Sunila Pradeep, ${ }^{1}$ Xianbin Yang, ${ }^{6}$ Monika Haemmerle, ${ }^{1}$ \\ Cristian Rodriguez-Aguayo, ${ }^{2,7}$ Archana S Nagaraja, ${ }^{1}$ Rajesha Rupaimoole, ${ }^{1}$ Emine Bayraktar, ${ }^{7}$ \\ Recep Bayraktar, ${ }^{7}$ Li Li, ${ }^{3}$ Takemi Tanaka, ${ }^{8}$ Wei Hu, ${ }^{1}$ Cristina Ivan, ${ }^{1}$ Kshipra M Gharpure, ${ }^{1}$ \\ Michael H. McGuire, ${ }^{1}$ Varatharasa Thiviyanathan, ${ }^{3,4}$ Xinna Zhang, ${ }^{1,2}$ Sourindra N. Maiti, ${ }^{9}$ \\ Nataliya Bulayeva, ${ }^{3}$ Hyun-Jin Choi, ${ }^{1}$ Piotr L. Dorniak, ${ }^{1}$ Laurence J.N. Cooper, ${ }^{9}$ Kevin P. Rosenblatt, ${ }^{10}$ \\ Gabriel Lopez-Berestein, ${ }^{2,7,11}$ David G. Gorenstein,, ${ }^{3,4,6}$ and Anil K. Sood ${ }^{1,2,11}$ \\ 'Department of Gynecologic Oncology and Reproductive Medicine, '²enter for RNA Interference and Non-Coding RNAs, \\ The University of Texas MD Anderson Cancer Center, Houston, Texas, USA. ${ }^{3}$ Institute of Molecular Medicine, \\ ${ }^{4}$ Department of Nanomedicine and Biomedical Engineering, The University of Texas Health Science Center, Houston, \\ Texas, USA. ${ }^{5}$ Department of Internal Medicine, University of Texas Medical Branch, Galveston, Texas, USA. ${ }^{6}$ AM \\ Biotechnologies, Houston, Texas, USA. 'Experimental Therapeutics, The University of Texas MD Anderson Cancer \\ Center, Houston, Texas, USA. ${ }^{8}$ Biomedical Research Center, University of Oklahoma Health Sciences Center, Oklahoma \\ City, Oklahoma, USA. ${ }^{9}$ Department of Pediatrics, The University of Texas MD Anderson Cancer Center, Houston, Texas, \\ USA. ${ }^{10}$ Companion DX Reference Lab, Houston, Texas, USA. ${ }^{11}$ Department of Cancer Biology, The University of Texas MD \\ Anderson Cancer Center, Houston, Texas, USA.
}

Current antiangiogenesis therapy relies on inhibiting newly developed immature tumor blood vessels and starving tumor cells. This strategy has shown transient and modest efficacy. Here, we report a better approach to target cancer-associated endothelial cells (ECs), reverse permeability and leakiness of tumor blood vessels, and improve delivery of chemotherapeutic agents to the tumor. First, we identified deregulated microRNAs (miRs) from patient-derived cancer-associated ECs. Silencing these miRs led to decreased vascular permeability and increased maturation of blood vessels. Next, we screened a thioaptamer (TA) library to identify TAs selective for tumor-associated ECs. An annexin A2-targeted TA was identified and used for delivery of miR106b-5p and miR30c-5p inhibitors, resulting in vascular maturation and antitumor effects without inducing hypoxia. These findings could have implications for improving vascular-targeted therapy.

Authorship note: L.S. Mangala, H. Wang, and D. Jiang contributed equally to this work.

Conflict of interest: D.G. Gorenstein and $X$. Yang have a financial interest in AM Biotechnologies, which has commercialized X-aptamer bead-based selection technologies.

Submitted: March 25, 2016 Accepted: September 13, 2016 Published: October 20, 2016

Reference information: JCI Insight. 2016;1(17):e87754. doi:10.1172/jci.insight.87754.

\section{Introduction}

Although angiogenesis plays a critical role in cancer growth and metastasis, current antiangiogenic therapies have shown only modest efficacy (1). This is thought to occur, in part, because of the development of hypoxia with prolonged treatment $(2,3)$. The induction of hypoxia is not surprising given the pronounced reduction in the number of blood vessels with prolonged treatment (4). Targeting hypoxia pathways such as HIF1 $\alpha$ (e.g., topotecan, temsirolimus) has resulted in improved efficacy of antiangiogenic drugs (5, 6). However, novel approaches to target the tumor vasculature are needed to maintain antitumor efficacy without increasing hypoxia and other deleterious effects.

MicroRNAs (miRs) have been shown to play a vital role in tumor development and angiogenic processes by modulating the expression of critical angiogenesis factors (7). Deregulation of miRs has been identified in many cancer types and is closely related to tumor progression and metastasis. However, the role of tumor endothelium-derived miRs in regulating tumor vascularization remains to be elucidated. To address this knowledge gap, we carried out systematic profiling of miRs in ovarian tumor ECs compared with normal ECs. We identified a set of miRs that regulate the integrity of tumor vasculature. In validation studies, we focused on miRs upregulated in tumor ECs and found that inhibiting these miRs reduced vas- 
cular permeability and increased maturation without induction of hypoxia. Delivery of the miR inhibitors was achieved using nanoparticles (NPs) decorated with thiophosphate backbone-modified aptamers (thioaptamers [TAs]) identified through cell-based SELEX (cell-SELEX) approaches with TAs.

\section{Results}

Altered miRs in tumor vasculature. To identify miRs with altered expression in tumor vasculature, we isolated ECs from human tissue samples of high-grade serous ovarian cancer $(n=3)$ and normal ovarian tissues $(n$ $=3$ ) and performed nanostring analysis (7). Array results showed that 5 miRs were markedly upregulated and $7 \mathrm{miRs}$ were downregulated in ovarian tumor ECs compared with normal ECs (Supplemental Table 1; supplemental material available online with this article; doi:10.1172/jci.insight.87754DS1). We selected the 3 most highly upregulated miRs (miR106b-5p, miR30c-5p, and miR141-3p) and validated the expression of these overexpressed miRs in tumor ECs using 3 independent patient samples. Consistent with our discovery data set, expression of these miRs was substantially higher in tumor ECs than in normal ECs (Figure 1A). Prior to carrying out functional experiments, we asked whether tumor-derived factors could induce expression of these miRs. Indeed, exposure to conditioned media (CM) from ovarian cancer cells resulted in increased levels of miR106b-5p, miR30c-5p, and miR141-3p in RF24 and G1S1 ECs. Similar results were observed when normal ECs were cocultured with SKOV3ip1 cancer cells (Figure 1B). Since VEGF is the dominant factor in blood vessel leakiness (1), we hypothesized that VEGF-induced leakiness could be promoted by upregulation of these miRs. We checked the expression of miR106b-5p and miR30c$5 \mathrm{p}$ in the presence of $10 \mathrm{ng}$ VEGF. Expression of both miRs was substantially increased (2-fold) upon treatment of RF24 ECs with VEGF at 48 hours (Supplemental Figure 1A). Silencing of either VEGFR1 or VEGFR2 receptors using specific siRNAs (Supplemental Figure 1B) in VEGF-treated ECs resulted in decreased expression of both miR106b-5p and miR30c-5p compared with control cells (not exposed either to VEGF or CM), suggesting the involvement of VEGF-mediated upregulation of these miRs (Supplemental Figure 1C). Similar results were observed when normal ECs were exposed to CM after silencing of VEGFR1 and VEGFR2 receptors (Supplemental Figure 1C).

To determine the potential effects of deregulated miRs on angiogenesis, we used integrated pathway analysis and found that tight junction signaling was the pathway most affected by deregulated miRs (Supplemental Figure 2 and Supplemental Table 2). To confirm this finding, we analyzed the expression of several tight junction genes when these miRs were upregulated. Consistent with the pathway analysis results, exposure to tumor $C M$ resulted in decreased expression of several genes that regulate tight junctions, including magi2, mpdz (MUPP1), myh11 (myosin), claudin 4, claudin 5, cdh5 (VE-cadherin), occludin, zo1, and $z o 2$ in ECs (Figure 1C).

Because tight junction proteins play a crucial role in regulating the paracellular permeability of both epithelial cells and ECs, we next tested the effects of inhibiting miR106b-5p, miR30c-5p, and miR141-3p on these tight junction genes. In G1S1 cells, treatment with miR inhibitors resulted in $80 \%-85 \%$ lower miR levels at 36 hours (Supplemental Figure 3) and increased the expression of tight junction genes (Figure 1D). Because downregulation of tight junction proteins leads to increased permeability and angiogenesis, we analyzed the effect of miR silencing on endothelial permeability. Compared with untreated ECs, cells with silenced miR106b-5p, miR30c-5p, or miR141-3p showed a 57\%, 47\%, or 35\% decrease in endothelial permeability, respectively (Figure 1E). We next asked whether these miR inhibitors could have direct effects on endothelial tube formation. None of the $3 \mathrm{miR}$ inhibitors had a significant effect on endothelial tube formation in 3-dimensional assays (Figure 1F), indicating that their role is likely to be more important for the structural integrity of vessels rather than endothelial recruitment.

Based on these in vitro findings, we sought to determine the biological effects of these miRs on tumor growth and angiogenesis in vivo. Because miR106b-5p and miR30c-5p inhibition led to the greatest reduction in endothelial permeability, we tested these $2 \mathrm{miR}$ inhibitors in vivo. For specific delivery of miR inhibitors to tumor vasculature, we first developed a method for targeted delivery using TAs.

Targeted delivery to tumor vasculature. To develop a delivery system to target tumor vasculature, we used a large TA library. Freshly isolated human ovarian tumor ECs from 10 patients with ovarian cancer were used as target cells. We started the selection by screening a 73-mer single-stranded DNA TA library, containing a 30-mer random region flanked with 21- and 23-mer primers. Before incubating the TA library with the target cells, we first depleted it of all TAs that bind to normal ovarian ECs in a counter-selection step. The correct size of the TA library was confirmed using polyacrylamide gel electrophoresis after eluting the TAs from target cells and sepa- 

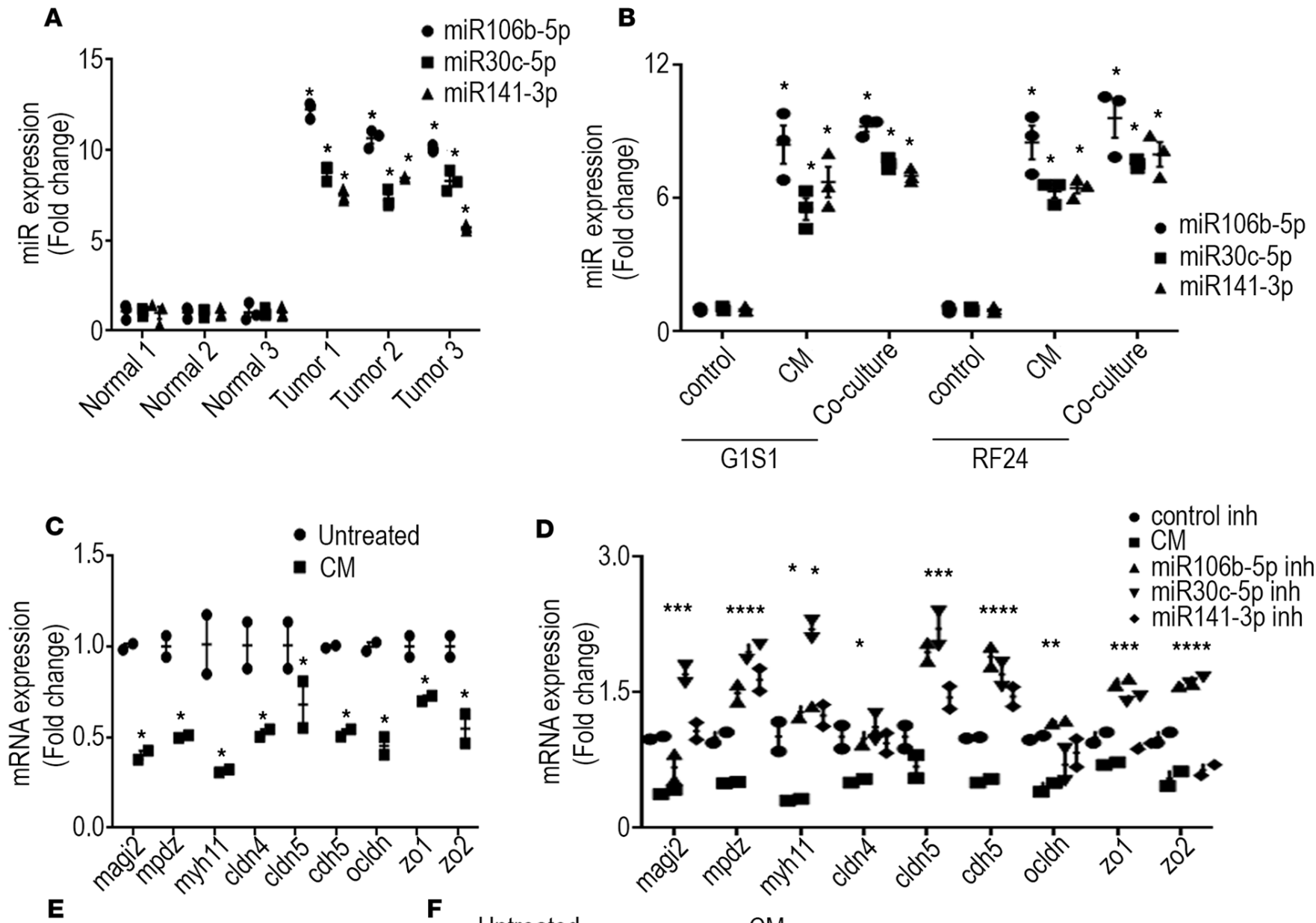

E
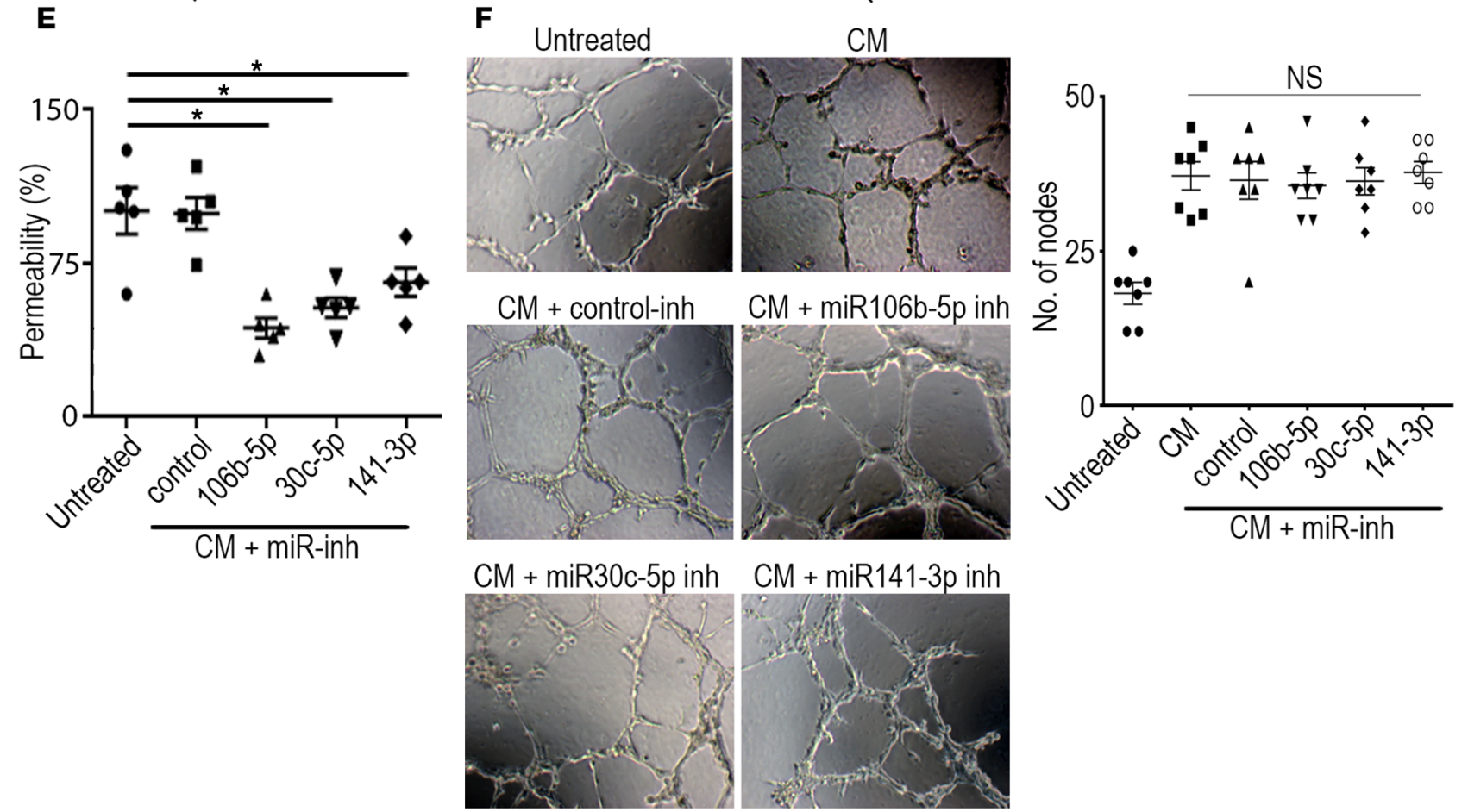
Figure 1. Upregulation of miRs in tumor endothelial cells decreases the expression of tight junction proteins. (A) Expression of miRs upregulated in patient tumor endothelial cells (ECs) compared with normal ovarian ECs. (B-E) ECs were incubated with conditioned medium (CM) from ovarian cancer SKOV3ip1 cells to mimic the tumor microenvironment. (B) Expression of miRs upregulated in tumor-associated ECs compared with untreated ECs. (C) Increased expression of miRs resulted in reduced levels of tight junction proteins. (D) miR silencing increased the expression of tight junction proteins. Fold changes in A-D represent the mean of triplicate experiments compared with normal or control or untreated cells. (E) miR silencing decreases the permeability in tumor-associated ECs. miR-inh, miRNA inhibitor. (F) Effect of miR silencing on angiogenesis (tube formation). All experiments were performed 3 times with triplicate samples $(n=3)$. Values are means \pm SEM. ${ }^{*} P<0.05$ (1-way ANOVA followed by a Tukey's multiple comparison post-hoc test). NS, not significant.

rating the TAs using streptavidin magnetic beads in each round of selection. Enrichment of the TAs that bound to ovarian cancer ECs was performed by gradually decreasing the number of tumor ECs in the selection process. Selection was completed after 10 rounds, and the TAs from rounds 4, 7, and 10 were cloned into a TOPO-TA cloning vector and sequenced, resulting in 45 sequences (Figure 2A). The secondary structures and free energies of folding were predicted by M-fold (8). Based on sequence homology, secondary structure, and low predicted folding free energies, a representative subset of 7 TA sequences were synthesized for binding tests (Supplemental Figure 4A). Seven Cy3-labeled TAs were examined for specific binding to human ovarian cancer tissues relative to a negative random control, TA-R4. Based on the fluorescence intensity of the Cy3-labeled TAs bound to the tumor vasculature, Endo28 and Endo31 exhibited strong binding affinity for tumor ECs (Supplemental Figure 4B). The Cy3-labeled Endo28 showed increased binding to tumor vasculature compared with normal vasculature (Figure 2B). Similarly, Endo28 did not show specific binding to other cell types including macrophages (Supplemental Figure 4C) and smooth muscle cells (Supplemental Figure 4D).

To identify the targeted surface proteins, we incubated human microvascular ECs (HMVECs) with biotinylated Endo28, Endo31, or R4 individually at $4^{\circ} \mathrm{C}$ and cross-linked the TAs with formaldehyde (9). The proteins cross-linked to the biotinylated TAs were collected by binding them to streptavidin magnetic beads and the proteins were subsequently proteolyzed on the beads. The resulting peptides were analyzed by mass spectrometry (10-12), which identified annexin A2 as the likely binding partner for both Endo28 and Endo31 (Supplemental Tables 3-5).

Immunofluorescence staining confirmed the increased expression of annexin A2 in human ovarian tumor vasculature compared with normal vasculature (Figure $2 \mathrm{C}$ ). Flow cytometry results indicated that annexin A2 was also expressed in ovarian cancer cell lines (data not shown) and proliferating HMVECs (Figure 2D). To determine the selectivity of annexin A2 as a binding partner of TAs, we silenced annexin A2 in HMVECs using a specific siRNA sequence. Transfection of HMVECs with a targeted siRNA sequence resulted in significant reduction of annexin A2 mRNA levels (Supplemental Figure 5A) and protein expression (Supplemental Figure 5B). Knockdown of annexin A2 resulted in greatly reduced binding of both Cy3-Endo28 and Cy3-Endo31 to HMVECs (Figure 2E).

For targeted delivery of miRs, we used TA-labeled chitosan (CH)-NPs. Following loading of control siRNA with $\mathrm{CH}$ using sodium triphosphate, we successfully conjugated TAs (R4 and Endo28) to the NPs by covalently coupling carboxyl groups to primary amines (Supplemental Figure 6, A-C). The physicochemical and stability characteristics of this NP system are presented in Supplemental Figure 6, D-H. The CH/Endo28-NPs were successfully internalized into RF24 and mouse ovarian ECs (MOECs) in a time-dependent manner (Supplemental Figure 7, A and B). Internalization of NPs decreased after incubation of MOECs with caveolin and clathrin inhibitors, suggesting that uptake of NPs into these cells occurs via both clathrin- and caveolin-dependent pathways (Supplemental Figure 8).

To test in vivo delivery, tumor-bearing (HeyA8) mice were injected with either control siRNA or Alexa 488-conjugated siRNA packaged into $\mathrm{CH} /$ Endo28-NPs and tumors were harvested 6 hours after NP injection. $\mathrm{CH} /$ Endo28-NPs bound to the vasculature (Figure 3A) and delivery of CH/Endo28-NPs with Alexa 488-conjugated siRNA were noted in more than $70 \%$ of the fields and examined after a single intravenous injection (Figure 3B). To determine whether annexin A2 expression is required for targeted delivery, we next evaluated the delivery of $\mathrm{CH} /$ Endo28-NPs into tumor ECs with and without annexin A2 silencing using a mouse annexin A2 siRNA sequence. The delivery of $\mathrm{CH} /$ Endo28-NPs into tumor ECs was substantially reduced, essentially to the level of delivery observed when using random TA-R4, after annexin A2 gene silencing (Figure 3C). A greater proportion of $\mathrm{CH} /$ Endo28-NPs was delivered to tumors than to any of the other organs (Supplemental Figure 9). We also used optical imaging to assess biodistribution; compared with controls (CH/R4-NPs), CH/ Endo28-NPs were largely taken up by the tumor, with minimal delivery to other organs such as the liver, lungs, kidneys, and spleen (Figure 3D). 
A

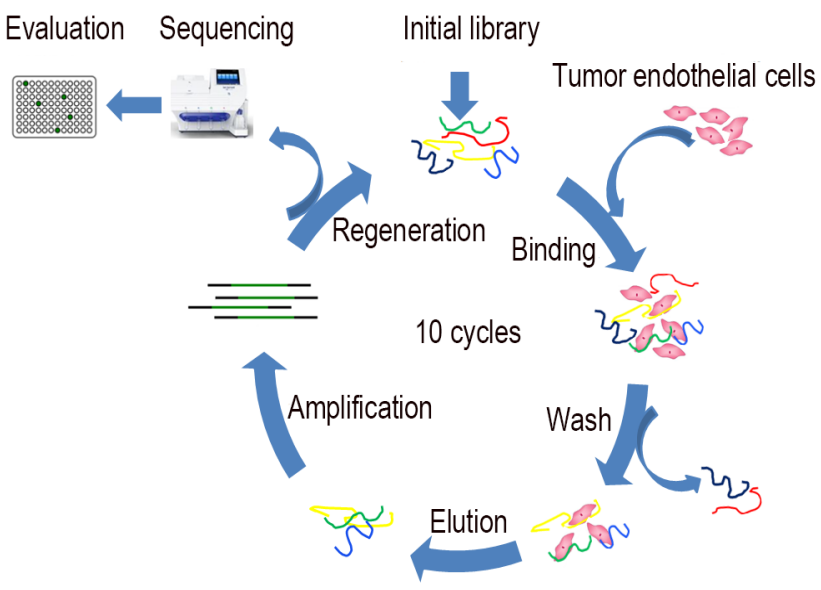

B

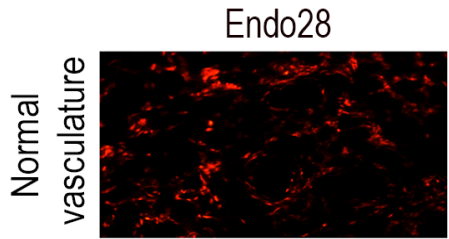

Endo28 +CD31
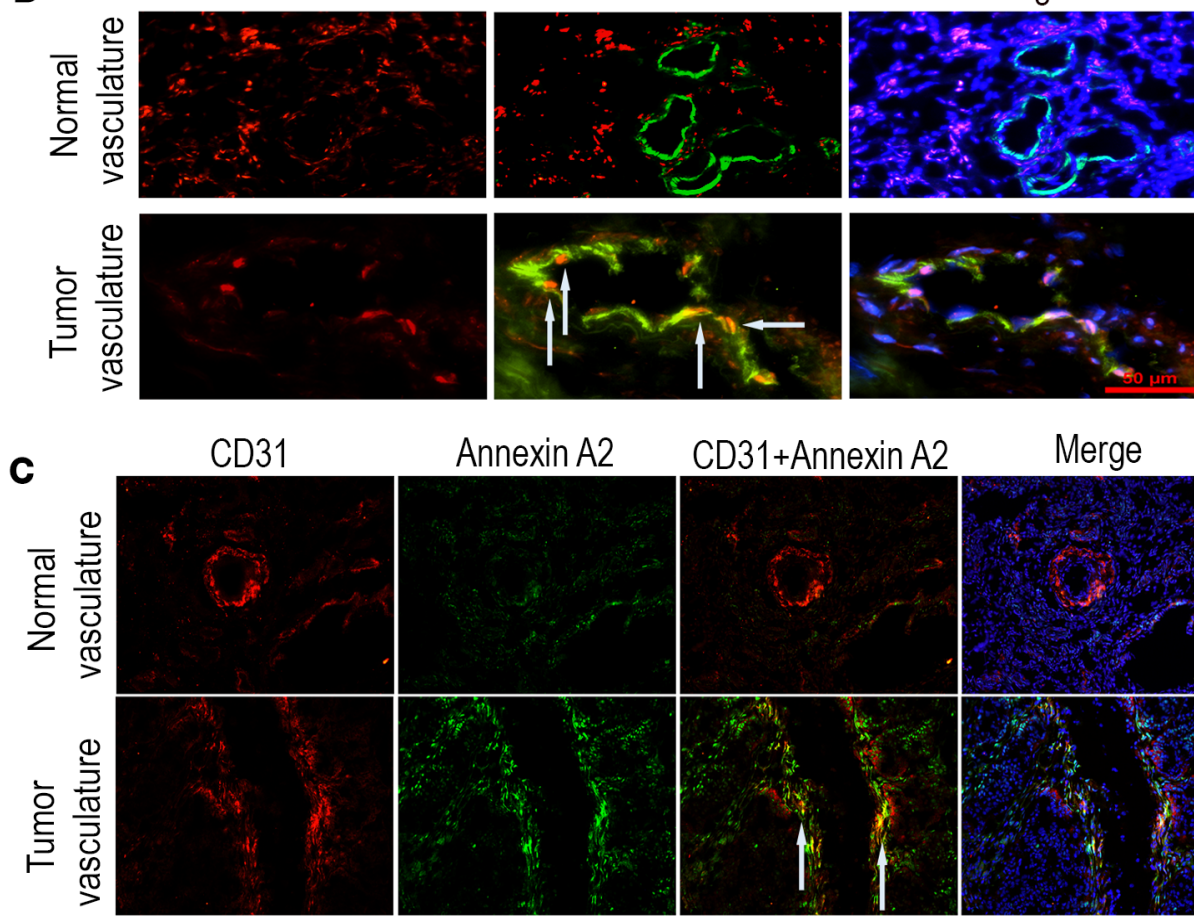

D

E

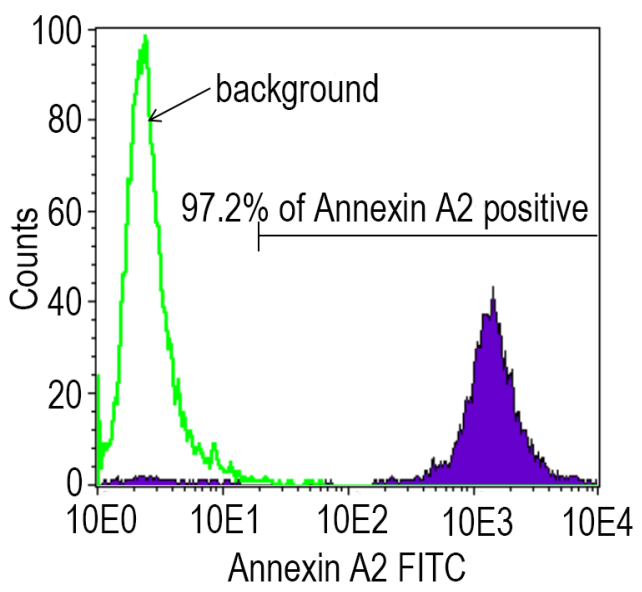

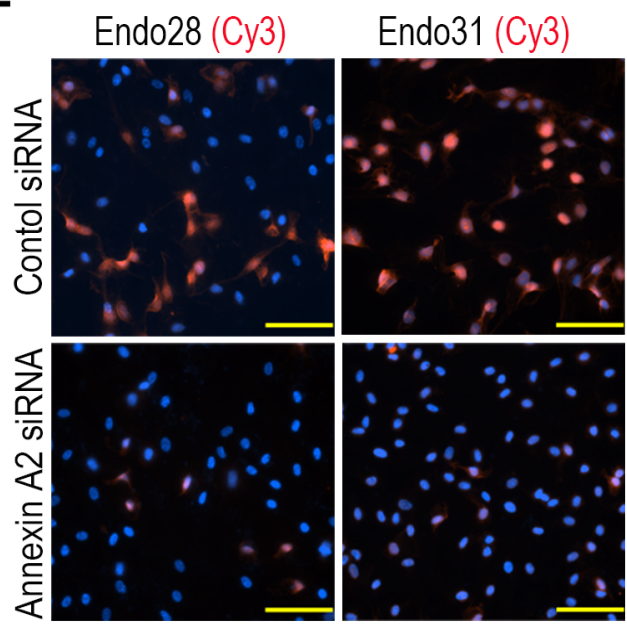

Figure 2. Selection and identification of thioaptamers that bind to human ovarian cancer vascular endothelial cells (ECs). (A) Cell-SELEX procedure with thioaptamers (TAs).

A library of ssDNA TAs was selected, and these TAs were incubated with purified human ovarian cancer ECs, washed, eluted, and amplified for 10 rounds. (B) Representative tumor sections showing binding of Endo28 to human ovarian tumor vasculature. Sections from normal ovary and tumor were incubated with Cy3-labeled Endo28 and anti-CD31 antibody to stain blood vessels. Nuclei are shown in blue, blood vessels in green, and Endo28 in red. Arrows denote colocalization of Endo28 (red) and CD31 (green). (C) Representative tumor sections showing expression of annexin $A 2$ on human ovarian tumor vasculature. Sections from normal ovary and tumor were incubated with anti-annexin $\mathrm{A} 2$ and anti-CD31 antibodies. Nuclei are stained blue, annexin $\mathrm{A} 2$ green, and blood vessels red. Arrows denote colocalization of CD31 (red) and annexin A2 (green). (D) Annexin A2-positive human microvascular ECs (HMVECs) were detected by flow cytometry. (E) Representative immunofluorescence staining showing that silencing of annexin $A 2$ resulted in reduced binding of Endo28 and Endo31 to HMVECs. Scale bars: $100 \mu \mathrm{m}$ (C and $\mathbf{E})$. 


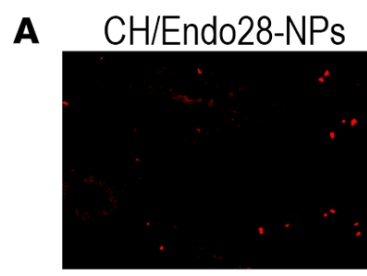

C
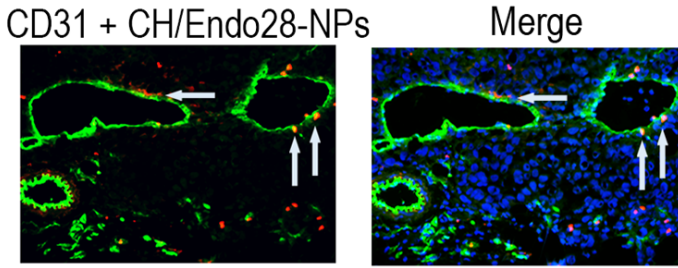

$\mathrm{CH} /$ Endo28-NPs

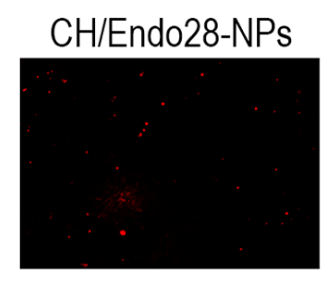

Alexa 488 siRNA
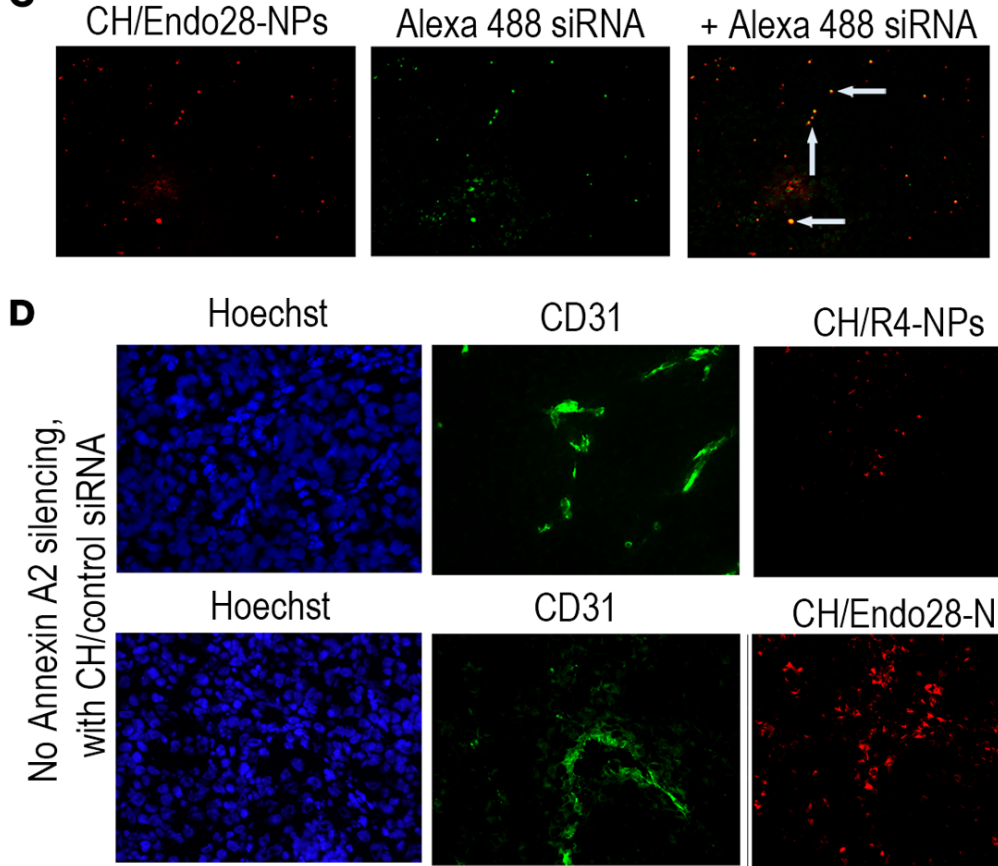

Hoechst
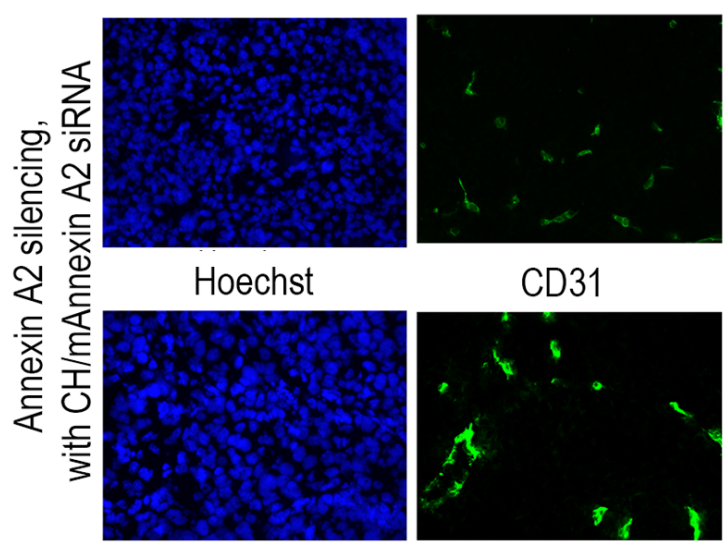

E
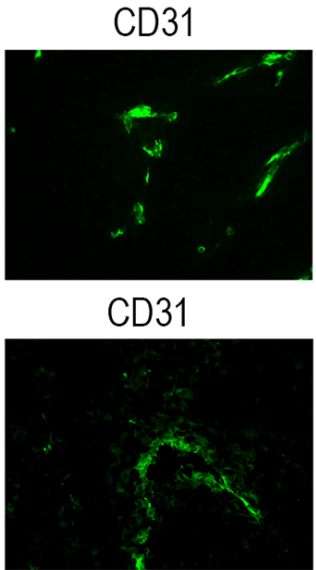

CD31

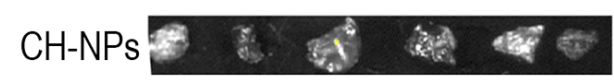

CH/R4-NPs

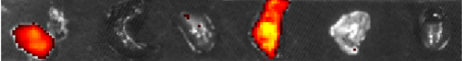

$\mathrm{CH} /$ Endo28-NPs

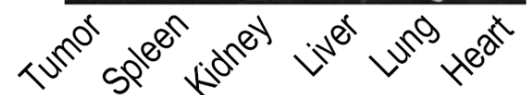

B

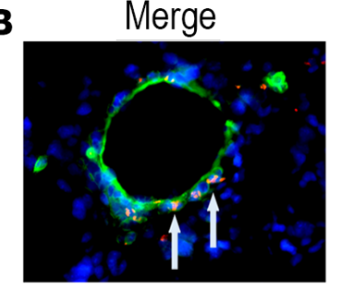

Merge

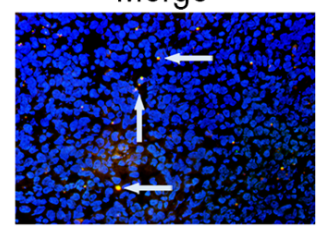

CH/R4-NPs

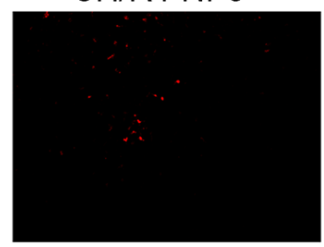

CH/Endo28-NPs

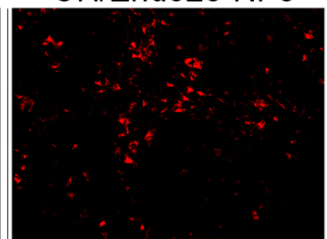

CH/R4-NPs

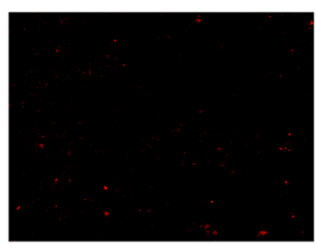

CH/Endo28-NPs
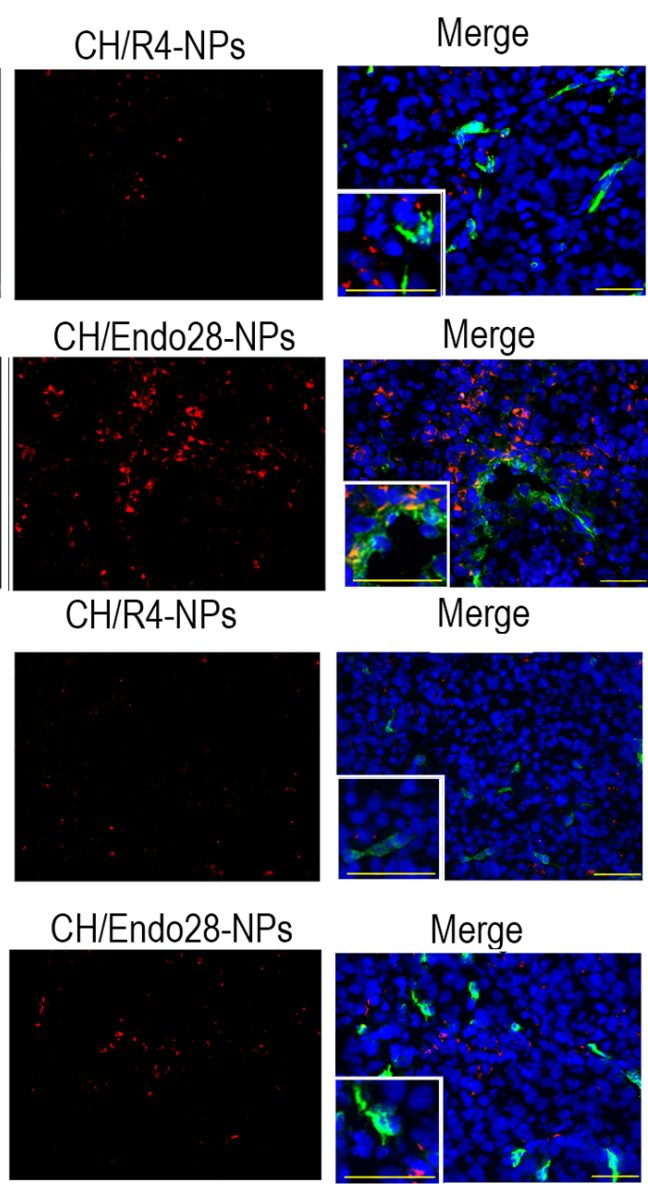

Merge

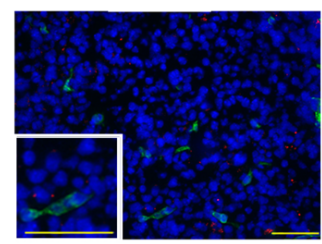

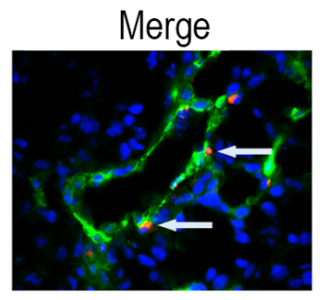

6.0 Epifluorescence

5.0

$-4.0 \times 10 E 6$

3.0 
Figure 3. Delivery of CH/Endo28-NPs to tumor vasculature. Tumors and organs were harvested 6 hours after a single intravenous injection of CH-Endo28-NPs (150 pmol/mouse) into HeyA8 tumor-bearing mice and stained with anti-CD31 antibody to detect blood vessels. (A) Representative tumor sections showing binding of $\mathrm{CH} / \mathrm{Endo28-NPs}$ to tumor vasculature. Nuclei are shown in blue, blood vessels in green, and $\mathrm{CH}-\mathrm{En}$ do28-NPs in red (Cy3). CH/Endo28-NPs, chitosan/Endo28-nanoparticles. (B) Representative tumor sections showing colocalization of CH-Endo28-NPs (red) with blood vessels (green). Arrows in A and B denote colocalization of Endo28 (red) and CD31 (green). (C) Representative tumor sections showing delivery of Alexa 488-conjugated siRNA using CH/Endo28-NPs into tumor cells (blue indicates nuclei, green indicates Alexa 488-conjugated siRNA, and red indicates CH-Endo28-NPs). CH/R4-NPs, chitosan/R4-nanoparticles. Arrows denote colocalization of CH/Endo28 (red) and Alexa 488 siRNA (green).(D) Representative tumor sections showing that annexin A2 silencing reduced delivery of $\mathrm{CH} / \mathrm{Endo28}-\mathrm{NPs}$ to the tumor vasculature. Mice were treated with either $\mathrm{CH} /$ control siRNA or $\mathrm{CH} /$ mouse annexin $\mathrm{A} 2$ ( $\mathrm{CH} / \mathrm{mAnnexin} \mathrm{A2)} \mathrm{siRNA} \mathrm{for} 48$ hours before a single intravenous injection of CH/R4-NPs or CH/Endo28-NPs. Tumors were harvested 24 hours after NP injection and stained with anti-CD31 antibody to visualize blood vessels. Scale bars: $50 \mu \mathrm{m}$. (E) Distribution of CH/Endo28-NPs in tumor cells and organs, examined using IVIS imaging (see Supplemental Methods). Optical imaging of organs and tumors from HeyA8 tumor-bearing mice treated with CH-NPs or CH/R4-NPs or CH/Endo28-NPs shows fluorescence intensity overlaid on white light images of different mouse organs and tumor.

Biological effects of miR106b-5p and miR30c-5p silencing. To evaluate the in vivo biological effects of miR106b-5p and miR30c-5p silencing, we used a well-characterized mouse model. Because HeyA 8 cancer cells have low expression of these 2 miRs (Supplemental Figure 10), we used this model to assess the influence of miRs on tumor vasculature. Treatment with $\mathrm{CH} /$ Endo28-miR106b-5p inhibitor resulted in $50 \%$ reduction in tumor growth, and treatment with $\mathrm{CH} /$ Endo28-miR30c-5p inhibitor resulted in $\sim 25 \%$ reduction in tumor growth (Figure $4, \mathrm{~A}-\mathrm{C}$ ). Remarkably, the addition of paclitaxel to either the miR106b-5p inhibitor or the miR30c-5p inhibitor resulted in a substantially greater reduction in tumor growth $(78 \%-90 \% ; P<0.05)$ compared with controls (Figure 4, A-C). Mean mouse body weights were similar regardless of the treatment and animal model (data not shown), suggesting that these combinations lack any overt toxicity.

To determine whether these miRs could have relevance for other tumor types, we examined the expression of annexin A2 in uterine (HEC1A) and breast (MDA-MB-231) tumor vasculature. Both tumors showed expression of annexin A2 (Supplemental Figure 11A). To evaluate the therapeutic efficacy of $\mathrm{CH} /$ Endo28-NPs, we next used an orthotopic breast cancer model (MDA-MB-231). Treatment with $\mathrm{CH} /$ Endo28-miR106b-5p inhibitor resulted in 51\% $(P<0.001)$ reduction in tumor growth and a combination of paclitaxel and miR106b-5p silencing resulted in greater than $90 \%$ reduction in tumor growth $(P<0.0001)$ compared with the control miR inhibitor group (Supplemental Figure 11, B-D).

Next, we examined the vascular characteristics of the treated tumors. Pericyte coverage was markedly increased in tumors treated with $\mathrm{miR}$ inhibitors compared with those treated with control miR inhibitor (Figure 4, D and G). Moreover, vascular leakiness was also decreased after miR silencing, suggesting that silencing these miRs stabilized blood vessels by reducing the leakiness (Figure 4, E and G). The hypoxia level was decreased after miR silencing (Figure 4F), which is consistent with improved vascular function. Similar effects were observed in combined treatment with miR inhibitors and paclitaxel (Figure 4, D-G). Neither of these miR inhibitors resulted in significant reduction of blood vessel number (Figure $4 \mathrm{H}$ ), which is consistent with their role in promoting vascular structure rather than EC viability. Expression of tight junction proteins such as zo1/2 and claudin 5 was markedly increased in tumor endothelium after miR silencing (Figure 5, A-C). No significant reduction was observed in the number of blood vessels after miR silencing (Supplemental Figure 12A). Given the profound antitumor effects with paclitaxel despite the lack of reduction in microvessel density, we asked whether the improved vascular features in the tumor microenvironment could lead to increased drug delivery. Indeed, inhibition of either miR increased the delivery of paclitaxel into tumors. Tumor-bearing animals treated with the CH/Endo28-miR106b-5p inhibitor showed 4-times higher paclitaxel content; those treated with the $\mathrm{CH} /$ Endo28-miR30c-5p inhibitor showed 2.5-times higher paclitaxel content than those treated with the $\mathrm{CH} /$ Endo28-control miR inhibitor (Supplemental Figure 12B). Collectively, we propose a model showing that miR silencing leads to restoration of tight junction function and in turn decreases angiogenesis (Figure 6).

Biosafety and efficacy of CH/Endo28-siRNA-NPs. We next evaluated the effect of CH/Endo28-NPs on C57/BL6 mice after intravenous administration of a therapeutic dose of $\mathrm{CH} /$ Endo28-control siRNA (5 $\mu \mathrm{g}$ of siRNA). The blood chemistry results confirmed that the NPs did not cause any significant changes in any of the parameters tested (Supplemental Figure 13, A-F). Similar to the blood chemistry results, the values of the cytokines tested were not significantly higher in the mice injected with NPs than in control mice. 


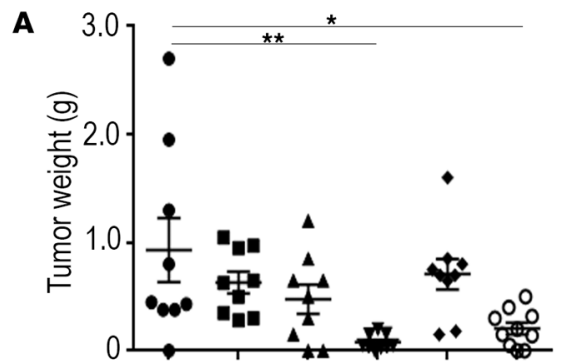

miR-inh control 106b-5p 30c-5p Paclitaxel

C

miR-inh

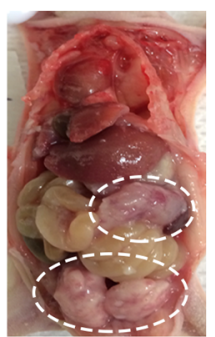

control

Paclitaxe

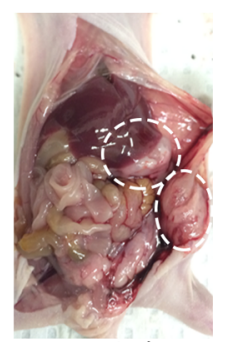

control
B

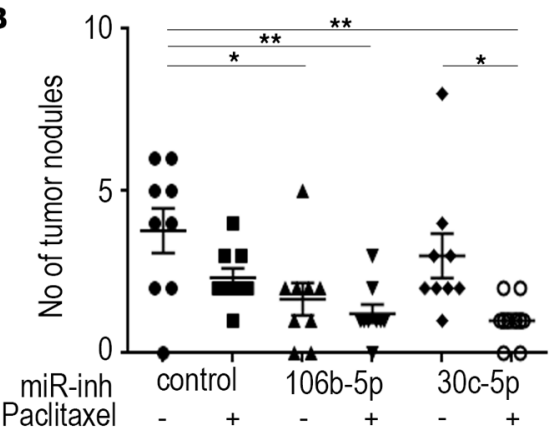

D

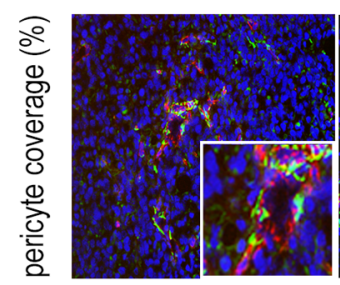

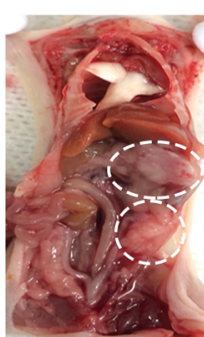

$106 b-5 p$

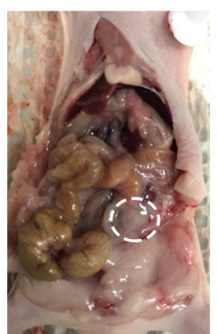

$106 \mathrm{~b}-5 \mathrm{p}$

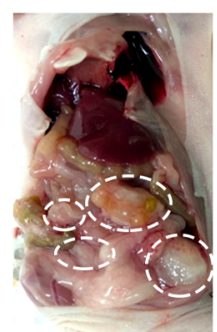

$30 c-5 p$

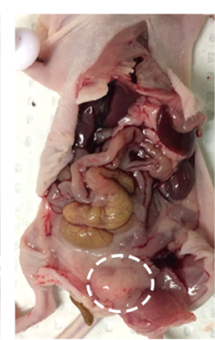

$30 c-5 p$
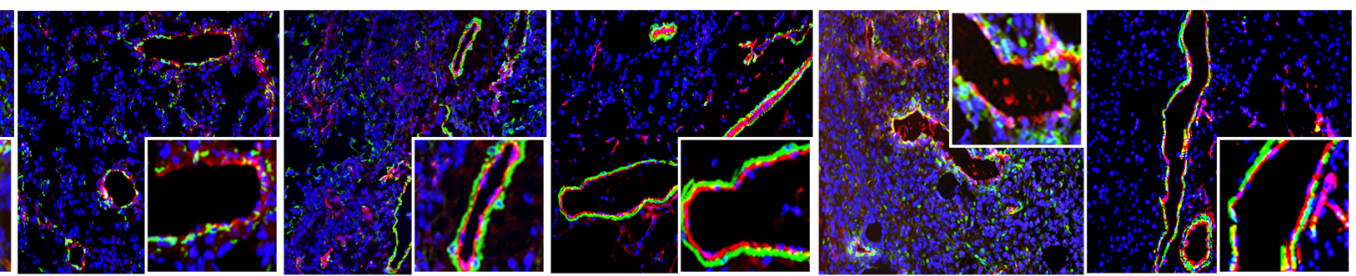

$\mathbf{E}$
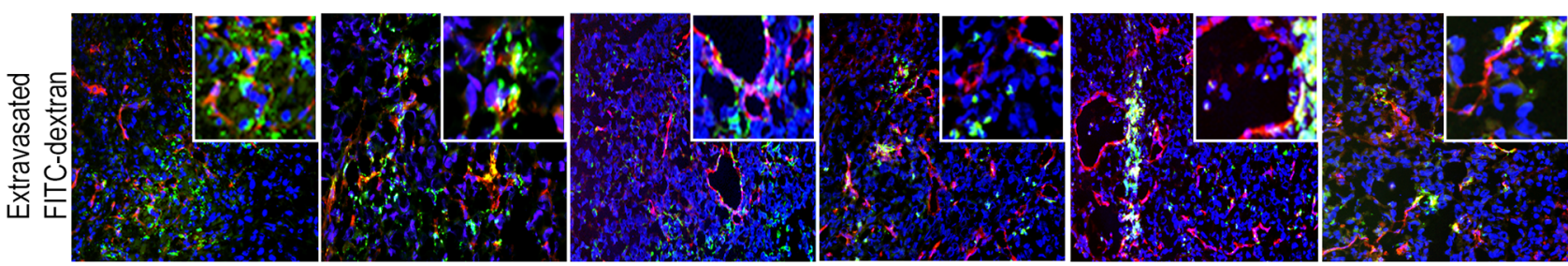

$\mathbf{F}$
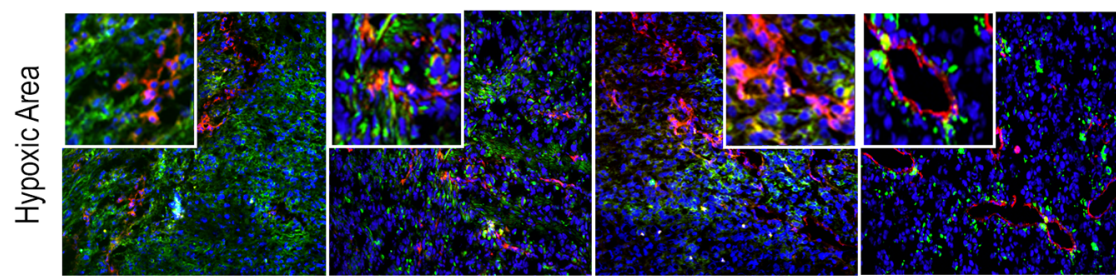

miR-inh

control

control

$106 b-5 p$

$106 b-5 p$
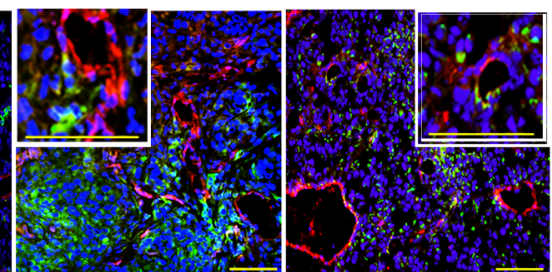

Paclitaxel

G

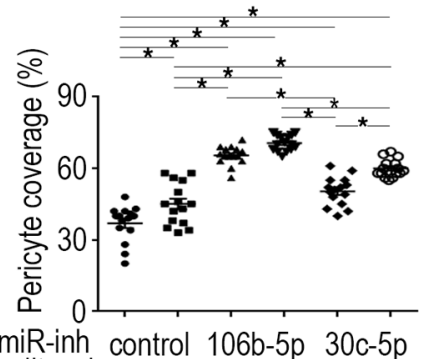

maclitaxe control 106b-5p 30c-5p

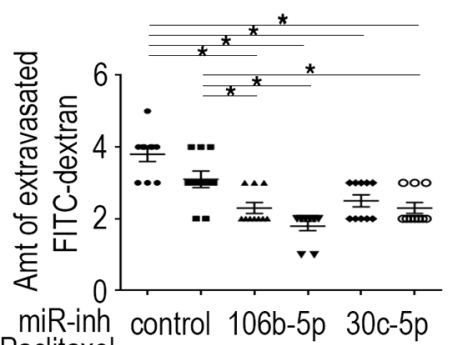

H

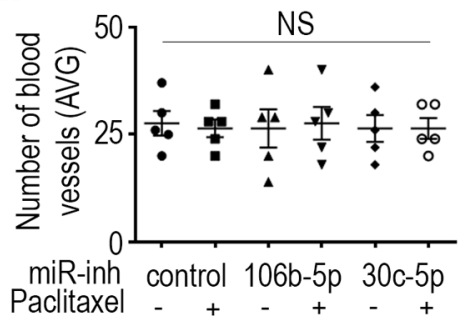


Figure 4. Therapeutic efficacy of CH/Endo28-NPs in a HeyA8 ovarian cancer orthotopic mouse model. Seven days following tumor cell injection, mice were randomly divided into 6 groups (9-10 mice per group) to receive 1 of the following 6 therapies: (i) CH/Endo28-control miR inhibitor, (ii) $\mathrm{CH} /$ Endo28-control miR inhibitor + paclitaxel, (iii) CH/Endo28-miR106b-5p inhibitor, (iv) CH/Endo28-miR106b-5p inhibitor + paclitaxel, (v) CH/Endo28miR30c-5p inhibitor, or (vi) CH/Endo28-miR30c-5p inhibitor + paclitaxel. Mice were sacrificed when any animals in a control or treatment group became moribund (after 3 to 4 weeks of therapy). (A) Mean tumor weight. (B) Mean number of tumor nodules. (C) Representative images of tumor burden from at least 5 mice per group. (D) Representative tumor sections showing effect of miR silencing on pericyte coverage. Tumor sections were stained with anti-desmin (pericyte coverage marker) and anti-CD31 (for blood vessels) antibodies respectively (red indicates blood vessels, green indicates pericyte coverage, and blue indicates nuclei). (E) Representative tumor sections showing effect of miR silencing on vascular permeability of blood vessels after i.v. injection of FITC-dextran. Tumor sections were stained with anti-CD31 antibody (red indicates blood vessels, green indicates FITC-dextran, and blue indicates nuclei). In $\mathbf{D}$ and $\mathbf{E}$, staining was done on 5 sections per group. (F) Determination of extent of hypoxia after staining tumor sections with hypoxia marker anti-CA9 and anti-CD31 antibodies (red indicates blood vessels, green indicates hypoxia, and blue indicates nuclei). (C) Quantification of pericyte coverage and extravasated FITC-dextran. Pericyte coverage was determined by the percentage of vessels with $\geq 50 \%$ coverage of desmin in positive cells in 5 random fields of each section at $\times 200$ magnification of each tumor. Quantification of extravasated FITC-dextran was carried out using fluorescence microscopy under the green fluorescent filter using the following scoring system: 0 points, no staining; 1 point, $<25 \% ; 2$ points, $25 \%-50 \%$; 3 points, 50\%-75\%; 4 points, $75 \%-100 \%$ FITC-dextran (using $8-10$ sections per tumor at $\times 200$ magnification. (H) Quantification of average number of blood vessels after indicated treatments. To quantify microvessel density, we recorded the number of blood vessels that stained positive for CD31 in 5 random fields of each section at $\times 200$ magnification for each sample. Values are the mean $\pm \mathrm{SEM}$. ${ }^{*} P<0.05$, ${ }^{* *} P<0.01$, (1-way ANOVA followed by a Tukey's multiple comparison post-hoc test). NS, not significant. Scale bars: $100 \mu \mathrm{m}$. CH., chitosan; miR-inh, miRNA inhibitor.

\section{Discussion}

In the current study, we developed a potentially new method of targeting tumor angiogenesis through 2 levels of targeting: (a) binding of TA-Endo28-decorated CH-NPs (CH/Endo28-NPs) to the tumor ECspecific membrane protein annexin $\mathrm{A} 2$, and (b) delivering miR inhibitors to the tumor vasculature and restoring tight junction function. We showed that this technique markedly improved the delivery of chemotherapeutic agents (i.e., paclitaxel) and reduced tumor growth in orthotopic ovarian cancer mouse models.

Despite promising results in preclinical models, approved antiangiogenic therapies using monoclonal antibodies have shown modest and transient efficacy in the clinic (13). Novel approaches to inhibit tumor angiogenesis in a specific and long-lasting manner are therefore critically needed. Targeting receptors specific to tumor ECs in combination with RNAi-mediated gene silencing provides a highly selective and effective new mode of antiangiogenic therapy. Several studies have shown that a combination of bevacizumab antibody and chemotherapy is required to improve survival in patients with non-small cell lung cancer, metastatic colorectal cancer, and HER2-negative breast cancer (14-17). Huang et al. showed that treatment with low doses of anti-VEGFR2 antibody helps restore the function of tumor vasculature, reducing leakiness and interstitial pressure, and thereby allowing for improved drug delivery in animal models (18). However, the periodic use of antiangiogenic antibodies leads to treatment resistance and ultimately tumor relapse. The technique developed in the current study helps overcome these challenges by selectively delivering antitumor agents directly to the tumor vasculature.

The differences between normal and tumor vasculature have been conventionally studied using cell culture and animal models. However, the vascular characteristics in these models may not resemble those in human tumors (19). In the current study, ECs were extracted directly from patient tumor samples to obtain a more accurate molecular profile of the tumor vasculature in human disease. Many of the top dysregulated miRs are directed toward tight junction modulation. Unsurprisingly, vascular remodeling is a process associated with tumor growth and metastasis. Increases in vessel permeability can be characterized as loss of endothelial cell-cell adhesion, as reflected by downregulation of important tight junction proteins such as occludin, claudins, zo1/2, and VE-cadherin. ECs are the first barrier that tumor cells must cross to enter the circulation for metastasis. Immature tumor vasculature and resultant high interstitial pressure contribute to an unfriendly microenvironment for chemotherapeutic drug delivery. Selective interference with these dysregulated miRs can globally restore endothelial tight junctions and enhance the effect of chemotherapy. A recent study has demonstrated that silencing of hypoxia-induced miR101 can reduce the number of capillaries in the ischemic hind limb and interfere with regional blood flow (20).

We also screened aptamers with high binding affinities for these patient-derived tumor ECs using the cell-SELEX method (10). The thiophosphate modifications in the TAs enhance both nuclease resistance and higher affinity for target proteins (21). Aptamers can possess dual functions, acting both as agents that target cell surface receptors or ligands, and as vehicles to deliver siRNA/miR cargo to specific cells. For example, a polyethylene glycol-conjugated aptamer that binds to VEGF has been approved by the US FDA for treatment of age-related macular degeneration (22), and more aptamers are currently being evaluated in clinical trials $(23,24)$. As a targeting moiety, a prostate-specific membrane antigen-targeting (PSMA-targeting) aptamer 

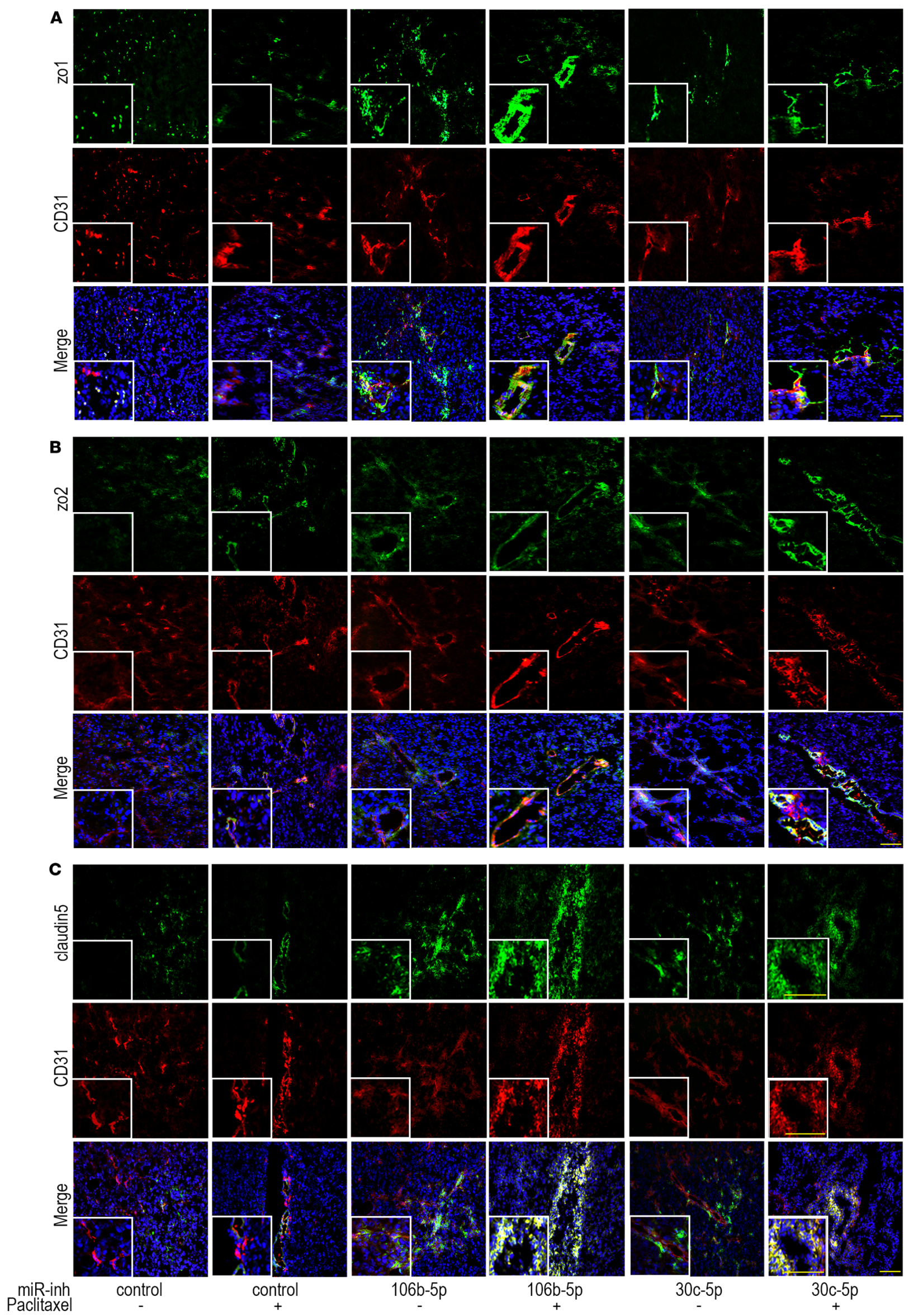
Figure 5. miR silencing increases the expression of tight junction proteins and chemotherapeutic drug delivery. Seven days following tumor cell injection, mice were randomly divided into 6 groups ( $9-10$ mice per group) to receive 1 of the following 6 therapies: (i) CH/Endo28-control miR inhibitor, (ii) CH/Endo28-control miR inhibitor + paclitaxel, (iii) CH/Endo28-miR106b-5p inhibitor, (iv) CH/Endo28-miR106b-5p inhibitor + paclitaxel, (v) CH/Endo28miR30c-5p inhibitor, or (vi) CH/Endo28-miR30c-5p inhibitor + paclitaxel. Mice were sacrificed when any animals in a control or treatment group became moribund (after 3 to 4 weeks of therapy). Tumor sections were stained with anti-zo1, -zo2 and -claudin 5 along with -CD31 antibodies. (A) Expression of zo1 (red indicates blood vessels, green indicates zo1). (B) Expression of zo2 (red indicates blood vessels, green indicates zo2). (C) Expression of claudin 5 (red indicates blood vessels, green indicates claudin 5). Staining was done on 5 sections per group (A-C). Scale bars: $100 \mu \mathrm{m}$. miR-inh, miRNA inhibitor.

has been conjugated to doxorubicin micelles, which led to an increased uptake by PSMA-positive CWR22Rnu1 prostate cancer cells and resulted in higher cytotoxicity than that achieved using nontargeting micelles (25). More recently, a similar strategy was used in constructing ${ }^{225} \mathrm{Ac}$-loaded liposomes to target and selectively kill PSMA-expressing cells such as LNCaP, Mat-Lu, and HUVEC using radiation (26). However, aptamers utilized in these studies were selected on the basis of binding to isolated surface markers or recombinant proteins, which might poorly reflect the native protein conformations owing to denaturing conditions during purification $(27,28)$. We used patient-derived ovarian tumor ECs to screen aptamers, and the annexin A2targeted TAs used in the current study offer the advantage of high binding affinity for surface markers in their native conformations. Although $\mathrm{CH}$ has not yet been approved by the FDA for drug delivery purposes, it has already been used in hemostasis products and has cleared many of the safety concerns about application in clinical settings (29-31). Therefore, our CH/TA-NPs constitute a uniquely safe and efficient delivery system for targeting tumor ECs and interfering with key angiogenic genes, providing a potentially new approach to selectively suppress tumor growth and angiogenesis. Furthermore, these methods of aptamer selection and NP assembly allow for rapid and highly specific development of new targeted therapies for cancer, paving the way for truly personalized cancer intervention.

\section{Methods}

Cell lines and culture. Maintenance of the human epithelial ovarian cancer cells and ECs is described in the supplemental methods (32).

TA selection and annexin $A 2$ identification. In brief (see supplemental methods), we used an ssDNA-TA library and a cell-SELEX method to identify a few dozen putative TAs that target human ovarian cancer ECs. These TAs $(50 \mathrm{nM})$ were tested for their relative binding affinity for ECs on ovarian cancer tissue sections. Annexin A2 protein was identified as the target of these selected TAs by cross-linking and mass spectrometry (12).

In vitro transfection. We silenced miRs in ECs using miR inhibitors and RNAifect reagent (Qiagen) as described in the supplemental methods. VEGFR1 and VEGFR2 were silenced in RF24 cells and annexin A2 in HMVECs was silenced with either control or target siRNAs using Lipofectamine 2000 reagent (Invitrogen) as described previously (33). The expression of annexin A2, miRs, and tight junction proteins was determined using quantitative real-time PCR and Western blot analyses as described previously (34, 35). After transfecting RF24 cells with miR inhibitors, we performed cell permeability and tube formation assays as described in the supplemental methods.

Synthesis and characterization of CH/Endo28-siRNA-NPs. The activation and conjugation of TAs (negative control R4 and targeted Endo28) on $\mathrm{CH}$ and the characterization of NPs are described in the supplemental methods. In brief, the grafting efficiency of Endo28 on CH-NPs and the size and zeta potential of the $\mathrm{CH}$ /Endo28-siRNA-NPs were measured. The grafting of Endo28 on $\mathrm{CH}$ was confirmed by proton NMR ( ${ }^{1} \mathrm{H}$ NMR) and the morphology of the $\mathrm{CH}$ /Endo28-NPs was determined using atomic force microscopy. The intact binding of TA-Endo28 and siRNA with $\mathrm{CH}$ and the stability of NPs were confirmed by gel retardation and a stability assay using agarose gel electrophoresis. The internalization and endosomal escape of CH/Endo28-NPs in MOECs and RF24 ECs were analyzed using confocal microscopy.

$T A$ binding to ovarian tumor vasculature and therapeutic efficacy of miRs in ovarian tumors. The binding of $\mathrm{CH} /$ Endo28-NPs to the tumor vasculature and the uptake of NPs by the tumor, organs, and macrophages are described in the supplemental methods. Cell permeability, microvascular density, pericyte coverage, and delivery of paclitaxel are also described in the supplemental methods.

Statistics. All statistical tests were performed using Excel and GraphPad Prism 6 software. Differences between groups were evaluated using 2-tailed Student's $t$ test or 1-way ANOVA, adjusting for multiple comparisons. Results are presented as the mean \pm SEM. For animal experiments, 10 mice were assigned 
Leaky blood vessels

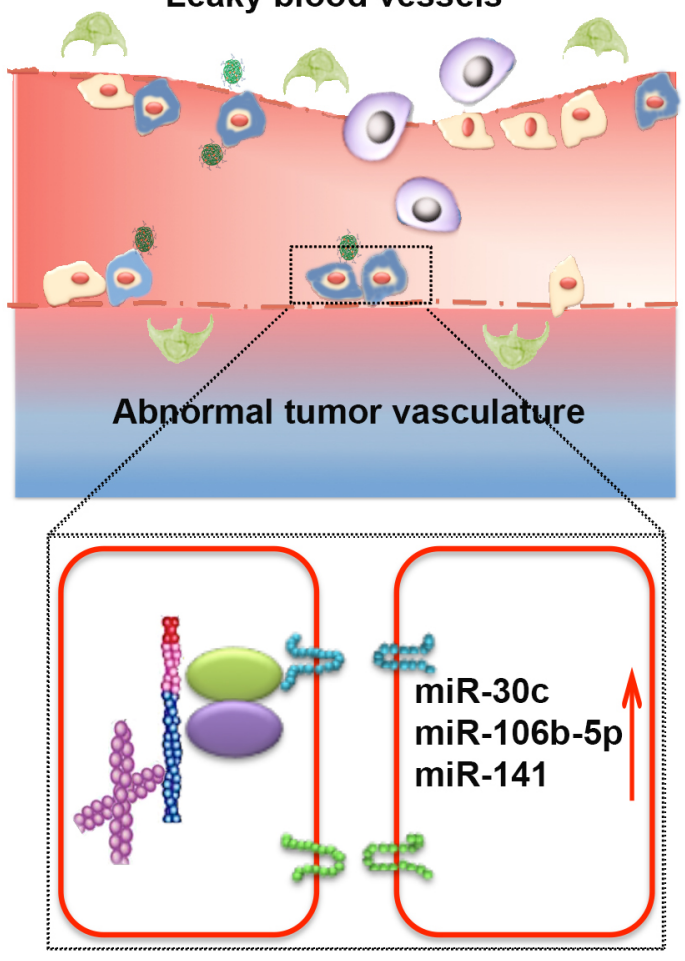

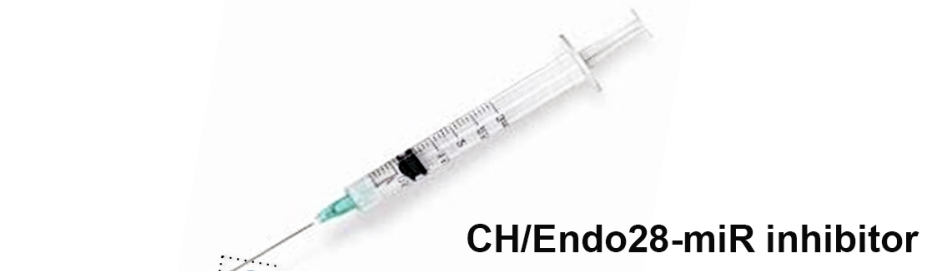

0
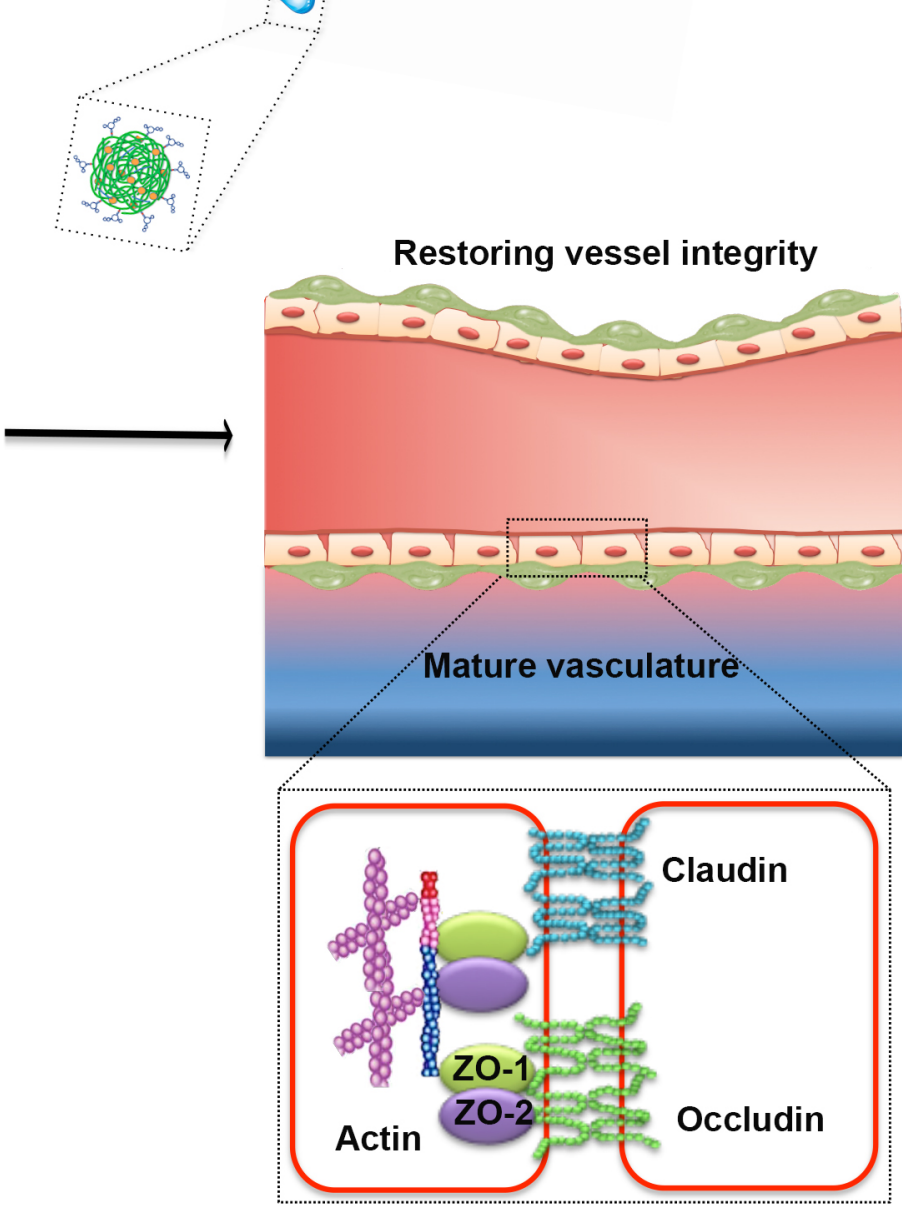

Figure 6. Proposed model of effects of miR silencing on restoring tight junction function and reducing vessel permeability.

per treatment group. This sample size gave $80 \%$ power to detect a $50 \%$ reduction in tumor weight with $95 \%$ confidence. Tumor weights and the number of tumor nodules for each group were compared using 1-way ANOVA followed by a Tukey's multiple comparison post-hoc test. For all statistical analyses, $P<0.05$ was considered statistically significant.

Study approval. All approvals for experiments depicted here were obtained from The University of Texas MD Anderson Cancer Center Institutional Review Board. All animal experiments were approved and supervised by the MD Anderson Cancer Center Institutional Animal Care and Use Committee.

\section{Author contributions}

LSM and DJ conceived and designed the experiments, synthesized and characterized the nanoparticles, conducted in vitro and in vivo experiments, and prepared the manuscript. HW conceived and designed the experiments; conducted in vitro cell and tissue binding, selection, and aptamer pull-down experiments; and prepared the manuscript. SYW and XZ conducted in vivo experiments. WH conducted isolation of endothelial cells. AS, XL, and TT conducted cell-SELEX experiments. NB and LL conducted mass spectrometry. SNM and LJNC conducted nanostring analysis. DEV, LGR, and VT provided thioaptamers. XY, 
provided 2'-OMe-PS2-modified anti-miR inhibitors. SP, CRA, EB, RB, ASN, RR, KMG, MHM, MH, HJC, RAP, and PLD assisted with in vivo experiments. HJC assisted in drawing the blood. KPR, GLB, DGG, and AKS conceived the project and provided guidance.

\section{Acknowledgments}

This work was supported by the Welch Foundation (AU-1296), National Institutes of Health (grants U54CA151668, CA109298, P50CA083639, P50CA098258, UH3TR000943, HHSN268201000037C), National Center for Advanced Translational Sciences Clinical and Translational Science Award (UL1 TR000371), the Ovarian Cancer Research Fund, Inc. (Program Project Development Grant), the Blanton-Davis Ovarian Cancer Research Program, the RGK Foundation, the Frank McGraw Memorial Chair in Cancer Research, the V-Foundation, and CPRIT grant RP110595.

Address correspondence to: Anil K. Sood, Department of Gynecologic Oncology and Reproductive Medicine, The University of Texas MD Anderson Cancer Center, Unit 1362, PO Box 301439, Houston, Texas 77030, USA. Phone: 713.745.5266; E-mail: asood@mdanderson.org.

1. Carmeliet P, Jain RK. Principles and mechanisms of vessel normalization for cancer and other angiogenic diseases. Nat Rev Drug Discov. 2011;10(6):417-427.

2. Rupaimoole R, et al. Hypoxia-mediated downregulation of miRNA biogenesis promotes tumour progression. Nat Commun 2014;5:5202.

3. Bottsford-Miller JN, Coleman RL, Sood AK. Resistance and escape from antiangiogenesis therapy: clinical implications and future strategies. J Clin Oncol. 2012;30(32):4026-4034.

4. Sennino B, McDonald DM. Controlling escape from angiogenesis inhibitors. Nat Rev Cancer. 2012;12(10):699-709.

5. Zighelboim I, et al. Multicenter phase II trial of topotecan, cisplatin and bevacizumab for recurrent or persistent cervical cancer. Gynecol Oncol. 2013;130(1):64-68.

6. Moroney JW, et al. A phase I trial of liposomal doxorubicin, bevacizumab, and temsirolimus in patients with advanced gynecologic and breast malignancies. Clin Cancer Res. 2011;17(21):6840-6846.

7. Wu SY, et al. A miR-192-EGR1-HOXB9 regulatory network controls the angiogenic switch in cancer. Nat Commun. 2016;7:11169.

8. Zuker M. Mfold web server for nucleic acid folding and hybridization prediction. Nucleic Acids Res. 2003;31(13):3406-3415.

9. Carey MF, Peterson CL, Smale ST. Chromatin immunoprecipitation (ChIP). Cold Spring Harb Protoc. 2009;2009(9):pdb.prot5279.

10. Tang Z, et al. Selection of aptamers for molecular recognition and characterization of cancer cells. Anal Chem. 2007;79(13):4900-4907.

11. Dua P, Kang HS, Hong SM, Tsao MS, Kim S, Lee DK. Alkaline phosphatase ALPPL-2 is a novel pancreatic carcinoma-associated protein. Cancer Res. 2013;73(6):1934-1945.

12. Mallikaratchy P, Tang Z, Kwame S, Meng L, Shangguan D, Tan W. Aptamer directly evolved from live cells recognizes membrane bound immunoglobin heavy mu chain in Burkitt's lymphoma cells. Mol Cell Proteomics. 2007;6(12):2230-2238.

13. Ferrara N, Hillan KJ, Gerber HP, Novotny W. Discovery and development of bevacizumab, an anti-VEGF antibody for treating cancer. Nat Rev Drug Discov. 2004;3(5):391-400.

14. Hurwitz H, et al. Bevacizumab plus irinotecan, fluorouracil, and leucovorin for metastatic colorectal cancer. $N$ Engl J Med. 2004;350(23):2335-2342.

15. Sandler A, et al. Paclitaxel-carboplatin alone or with bevacizumab for non-small-cell lung cancer. N Engl J Med. 2006;355(24):2542-2550.

16. Bear HD, et al. Bevacizumab added to neoadjuvant chemotherapy for breast cancer. NEngl J Med. 2012;366(4):310-320

17. von Minckwitz G, et al. Neoadjuvant chemotherapy and bevacizumab for HER2-negative breast cancer. $N$ Engl J Med. 2012;366(4):299-309.

18. Huang $\mathrm{Y}$, et al. Vascular normalizing doses of antiangiogenic treatment reprogram the immunosuppressive tumor microenvironment and enhance immunotherapy. Proc Natl Acad Sci U S A. 2012;109(43):17561-17566.

19. McGuire TF, Sajithlal GB, Lu J, Nicholls RD, Prochownik EV. In vivo evolution of tumor-derived endothelial cells. PLoS One. 2012;7(5):e37138.

20. Kim JH, et al. Hypoxia-responsive microRNA-101 promotes angiogenesis via heme oxygenase-1/vascular endothelial growth factor axis by targeting cullin 3. Antioxid Redox Signal. 2014;21(18):2469-2482.

21. Mann AP, et al. Identification of thioaptamer ligand against E-selectin: potential application for inflamed vasculature targeting. PLoS One. 2010;5(9):e13050.

22. VEGF Inhibition Study in Ocular Neovascularization (V.I.S.I.O.N.) Clinical Trial Group, et al. Year 2 efficacy results of 2 randomized controlled clinical trials of pegaptanib for neovascular age-related macular degeneration. Ophthalmology. 2006;113(9):1508.e1-1508.25.

23. Rosenberg JE, et al. A phase II trial of AS1411 (a novel nucleolin-targeted DNA aptamer) in metastatic renal cell carcinoma. Invest New Drugs. 2014;32(1):178-187.

24. Cataland SR, et al. Initial experience from a double-blind, placebo-controlled, clinical outcome study of ARC1779 in patients with thrombotic thrombocytopenic purpura. Am J Hematol. 2012;87(4):430-432. 
25. Xu W, et al. Aptamer-conjugated and doxorubicin-loaded unimolecular micelles for targeted therapy of prostate cancer. Biomaterials. 2013;34(21):5244-5253

26. Bandekar A, Zhu C, Jindal R, Bruchertseifer F, Morgenstern A, Sofou S. Anti-prostate-specific membrane antigen liposomes loaded with 225Ac for potential targeted antivascular $\alpha$-particle therapy of cancer. J Nucl Med. 2014;55(1):107-114.

27. Lupold SE, Hicke BJ, Lin Y, Coffey DS. Identification and characterization of nuclease-stabilized RNA molecules that bind human prostate cancer cells via the prostate-specific membrane antigen. Cancer Res. 2002;62(14):4029-4033.

28. Lefebvre BG, Gage MJ, Robinson AS. Maximizing recovery of native protein from aggregates by optimizing pressure treatment. Biotechnol Prog. 2004;20(2):623-629.

29. FDA allows marketing for first-of-kind dressing to control bleeding from certain battlefield wounds. Food and Drug Administration. http://www.fda.gov/newsevents/newsroom/pressannouncements/ucm391755.htm. Updated April 4, 2014. Accessed October 10, 2016

30. Brown MA, Daya MR, Worley JA. Experience with chitosan dressings in a civilian EMS system. J Emerg Med. 2009;37(1):1-7.

31. Millner RW, Lockhart AS, Bird H, Alexiou C. A new hemostatic agent: initial life-saving experience with Celox (chitosan) in cardiothoracic surgery. Ann Thorac Surg. 2009;87(2):e13-e14.

32. Lu C, et al. Regulation of tumor angiogenesis by EZH2. Cancer Cell. 2010;18(2):185-197.

33. Pecot CV, et al. Tumour angiogenesis regulation by the miR-200 family. Nat Commun. 2013;4:2427.

34. Cesarman GM, Guevara CA, Hajjar KA. An endothelial cell receptor for plasminogen/tissue plasminogen activator (t-PA). II Annexin II-mediated enhancement of t-PA-dependent plasminogen activation. J Biol Chem. 1994;269(33):21198-21203.

35. Brownstein C, et al. Annexin II mediates plasminogen-dependent matrix invasion by human monocytes: enhanced expression by macrophages. Blood. 2004;103(1):317-324. 